\title{
Long, Highly-Ordered High-Temperature Superconductor Nanowire Arrays
}

2008

Vol. 8, No. 11 3845-3849

\author{
Ke Xu and James R. Heath* \\ Division of Chemistry and Chemical Engineering, California Institute of Technology, \\ MC 127-72, Pasadena, California 91125
}

Received July 26, 2008; Revised Manuscript Received October 2, 2008

\begin{abstract}
The preparation and electrical properties of high-temperature superconductor nanowire arrays are reported for the first time. $\mathrm{YBa}_{2} \mathrm{Cu}_{3} \mathrm{O}_{7-\delta}$ nanowires with widths as small as $10 \mathrm{~nm}$ (much smaller than the magnetic penetration depth) and lengths up to $200 \mu \mathrm{m}$ are studied by four-point electrical measurements. All nanowires exhibit a superconducting transition above liquid nitrogen temperature and a transition temperature width that depends strongly upon the nanowire dimensions. Nanowire size effects are systematically studied, and the results are modeled satisfactorily using phase-slip theories that generate reasonable parameters. These nanowires can function as superconducting nanoelectronic components over much wider temperature ranges as compared to conventional superconductor nanowires.
\end{abstract}

A key question regarding the use of superconductors in nanoelectronic circuits is whether there is a critical size limit beyond which superconductivity can not be sustained. ${ }^{1-3} \mathrm{~A}$ superconducting wire becomes quasi-one-dimensional (quasi$1 \mathrm{D})$ when its width $(w)$ is comparable to or smaller than the material-dependent Ginzburg-Landau coherence length $\xi$ ( $>\sim 100 \mathrm{~nm}$ for elemental superconductors) and magnetic penetration depth $\lambda$ ( $\sim 40 \mathrm{~nm}$ for elemental superconductors). For bulk superconductors, the electrical resistance drops precipitously to zero below the superconducting transition temperature $\left(T_{\mathrm{c}}\right)$. For quasi-1D superconductors, the resistance decreases gradually below $T_{\mathrm{c}}$ due to phase-slip processes. ${ }^{2,4}$ This may result in resistive or insulating behaviors for thin $(\sim 10 \mathrm{~nm})$ wires when temperature approaches zero. ${ }^{1,2}$

Narrow width $(w \sim 10 \mathrm{~nm})$ nanowires $(\mathrm{NWs})$ made from elemental superconductors ${ }^{3,5-7}$ and from the binary alloy MoGe ${ }^{1,2,8}$ are all quasi-1D systems, and strong suppression of superconductivity by phase-slip processes has been observed in these systems. The very low $T_{\mathrm{c}}$ (typically below liquid helium temperature) limits their potential applications as zero-electrical resistance conductors or active components in nanoelectronic circuits. ${ }^{8-12}$ In comparison, the short $\xi(\sim 1$ $\mathrm{nm})$ that characterizes high-temperature superconductor (HTS) materials ${ }^{13}$ may reduce the influence on superconductivity of phase slip processes in HTS NWs, and the expected significantly higher $T_{\mathrm{c}}$ should uniquely enable applications of HTS NW materials.

However, achieving high-temperature superconductivity in NWs requires achieving the correct stoichiometry and the correct perovskite-like crystal structures of the HTS materials. This renders many superconductor NW fabrication meth-

* Corresponding author. E-mail: heath@ caltech.edu. ods ${ }^{1,5,6,14,15}$ inapplicable, and so little has been reported in this area. Short HTS NWs $(w \sim 50 \mathrm{~nm})$ have been synthesized, but superconductivity was only tested through magnetization measurements of powders of these materials. ${ }^{16,17}$ Patterning HTS NWs from epitaxially grown HTS thin films is also challenging: HTS materials are unstable toward processing steps involving acid, water, or moderately elevated temperatures. In addition, for patterning, HTS films are resistive to directional dry etching, and no selective chemical dry etch for HTS materials has been reported. For physical dry etching, HTS films exhibit slower etching rates than typical etch-mask materials. As a result, HTS thin films have only been patterned into short submicron bridges, and property measurements of those bridges have yielded inconsistent results. ${ }^{18-22}$ Bonetti et al. reported substantial broadening of the transition temperature width and telegraph-like resistance fluctuations in a $w=250 \mathrm{~nm} \mathrm{YBa}_{2} \mathrm{Cu}_{3} \mathrm{O}_{7-\delta}$ (YBCO) bridge ${ }^{21}$ but their findings were not confirmed by others in similar or narrower bridges. ${ }^{18-20,22}$ Mikheenko et al. studied YBCO bridges of several different widths and observed broadening of transition temperature width in a $w$ $=500 \mathrm{~nm}$ bridge. ${ }^{22}$ The broadening was modeled with phaseslip theories, but unrealistic fitting parameters were obtained because $w>\lambda \gg \xi$, and also no clear trend was found as the width of the bridges was varied. In summary, no clear size effects have been studied for HTS bridges (or wires) in the $w \ll \lambda \sim 100 \mathrm{~nm}$ region.

We have significantly modified the superlattice nanowire pattern transfer (SNAP) technique to overcome these limitations. SNAP translates the atomic control over the film thicknesses within a thin film superlattice into control over the width and spacing of NWs. ${ }^{23-25}$ SNAP is utilized to 

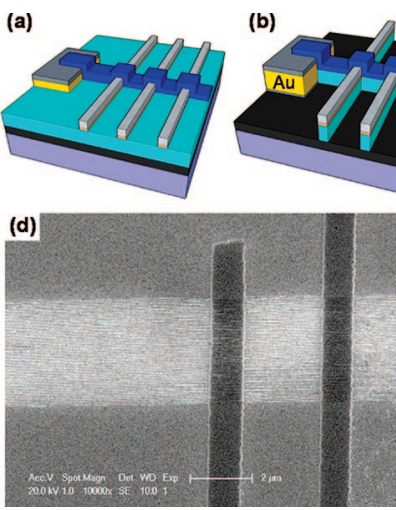
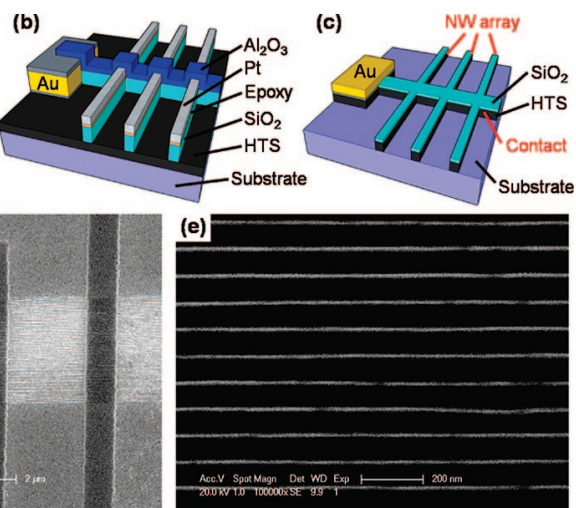

Figure 1. High-temperature superconductor nanowire arrays. $(\mathrm{a}-\mathrm{c})$ Process flow schematics. (a) Pt SNAP nanowires are deposited onto an $\mathrm{SiO}_{2}$-coated high-temperature superconductor (HTS) thin film. $\mathrm{Au}$-Ti metal pads are connected to the HTS layer far away from the nanowire array, and $\mathrm{Al}_{2} \mathrm{O}_{3}$ is lithographically patterned to define the contact electrodes. (b) The nanowires and contact patterns are translated into the $\mathrm{SiO}_{2}$ layer with selective reactive-ion etching. (c) The nanowires and contact patterns defined in the $\mathrm{SiO}_{2}$ layer are translated into the HTS film with $\mathrm{Ar}+\mathrm{O}_{2}$ physical dry etching. This also removes the top-lying Pt nanowires. The final structure is a single crystal HTS nanowire array device protected by a thin $\mathrm{SiO}_{2}$ film, with nanowires seamlessly connected to the contacts made from the same HTS film. (d) Scanning electron microscope image showing an $\mathrm{YBa}_{2} \mathrm{Cu}_{3} \mathrm{O}_{7-\delta}$ nanowire array and two contacts. Scale bar: $2 \mu \mathrm{m}$. (e) High-magnification micrograph of an $\mathrm{YBa}_{2} \mathrm{Cu}_{3} \mathrm{O}_{7-\delta}$ nanowire array (nanowires width $=10 \mathrm{~nm}$ ). Scale bar: $200 \mathrm{~nm}$.

stamp a NW array of refractory metal, such as Pt, onto the thin film from which the final NWs are produced. By using those Pt NWs as an etch mask, we previously prepared highquality elemental $(\mathrm{Nb})$ superconductor NWs. ${ }^{7}$ HTS materials are very unstable relative to $\mathrm{Nb}$. Thus, a water-free process that avoided temperatures above $120{ }^{\circ} \mathrm{C}$ was developed to accommodate the fragility of HTS thin films. Because HTS materials can only be patterned by slow physical dry etching ( $\sim 10$ times slower than the mask Pt NWs), a slow-etching $\mathrm{SiO}_{2}$ layer was sandwiched between the HTS thin film and the Pt NW array. Reactive-ion etching translated the Pt NW features into the $\mathrm{SiO}_{2}$ layer (Figure 1b), which then served as a mask for an $\mathrm{Ar}+\mathrm{O}_{2}$ physical dry etch of the HTS film (Figure 1c). $\mathrm{O}_{2}$ helped maintain the correct oxygen stoichiometry in the HTS material, ${ }^{26}$ and $\sim 10 \mathrm{~s}$ etch cycles were separated by $1 \mathrm{~min}$ cool-down periods to prevent heat accumulation within the HTS film.

HTS materials react with many metals and form highly resistive contacts. ${ }^{27}$ Thus, four-point probe contacts are made to the HTS NW array out of the same HTS film using a recently reported nanofabrication advance ${ }^{28}$ by patterning on top of the $\mathrm{SiO}_{2}$ layer before pattern translation of the Pt NWs (Figure 1a). Metal contacts for wirebonding are connected to the HTS layer far away from the NW array. Fabrication details are in the Supporting Information. See Figure 1d,e for images of YBCO NWs.

YBCO NW arrays of various NW dimensions were fabricated from a $30 \mathrm{~nm} c$-axis oriented epitaxial YBCO film (THEVA, Ismaning, Germany). Three $10 \mu \mathrm{m}$ wide strips of YBCO films underwent the same fabrication and testing as
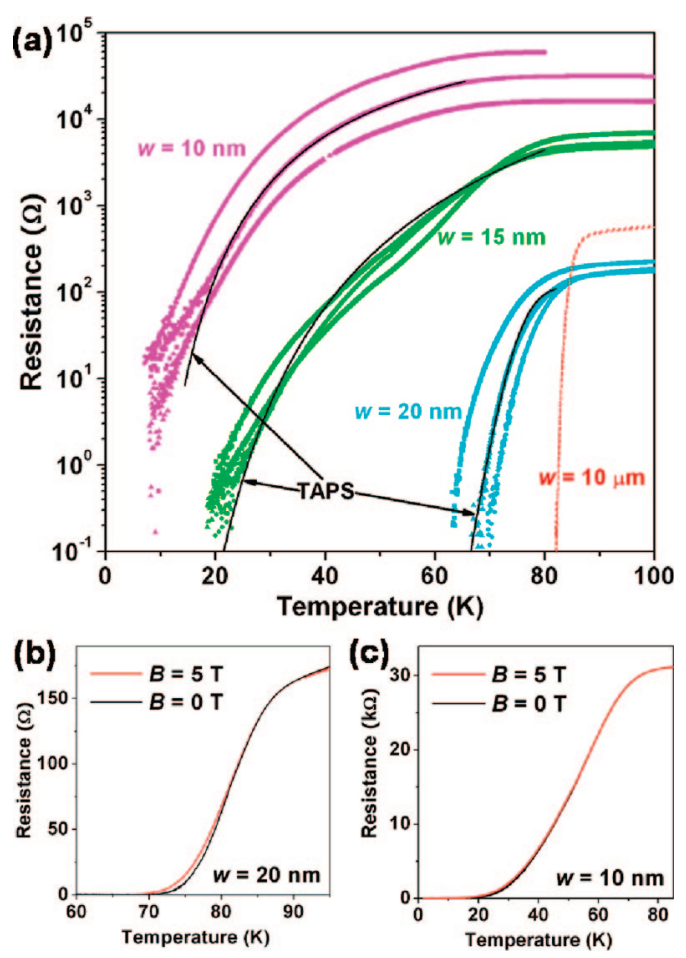

Figure 2. Temperature and magnetic field dependence for the fourpoint resistance of $\mathrm{YBa}_{2} \mathrm{Cu}_{3} \mathrm{O}_{7-\delta}$ nanowire arrays in the low-current limit. (a) Temperature dependence of resistance for nanowire arrays and films with various dimensions at zero magnetic field. Pink: arrays of 100 nanowires, width $w=10 \mathrm{~nm}$ and length $L$ (from top to bottom $)=200,100$, and $50 \mu \mathrm{m}$. Green: arrays of 150 nanowires, $w=15 \mathrm{~nm}$ and $L=35,30$, and $27 \mu \mathrm{m}$. Light blue: three arrays of 400 nanowires each, with $w=20 \mathrm{~nm}$ and $L=5 \mu \mathrm{m}$. Red dot lines: three geometrically identical strips fabricated through the same process, $w=10 \mu \mathrm{m}$ and $L=50 \mu \mathrm{m}$. Black lines are fits of the nanowire data to thermally activated phase slip models. (b) The resistance-temperature relationship for a 400 nanowire array with $w=20 \mathrm{~nm}$ and $L=5 \mu \mathrm{m}$, with and without an applied $5 \mathrm{~T}$ magnetic field. (c) The resistance-temperature relationship for an array of 100 nanowires with width $w=10 \mathrm{~nm}$ and $L=100 \mu \mathrm{m}$, with and without an applied $5 \mathrm{~T}$ magnetic field.

the NWs, and independent electrical measurements of these strips produced overlying resistance-temperature $(R-T)$ curves (Figure 2a). The strips exhibit a $T_{\mathrm{c}}$ of $85 \mathrm{~K}$, a sharp transition width of $\sim 3 \mathrm{~K}$, and a critical current density of $0.83 \mathrm{MA} / \mathrm{cm}^{2}$ at $77 \mathrm{~K}$. These values agree with those from the starting film. Thus the different behaviors observed in the NWs are attributed to size effects.

$R-T$ data, taken in the low-current limit $(\sim 1 \mathrm{nA}$ per NW) (Figure 2), reveals that high-temperature superconductivity is retained in all NWs, as characterized by a significant drop in resistance when $T$ is reduced below $T_{\mathrm{c}} \sim 80 \mathrm{~K}$. Consistent trends are found for NWs with the same widths, whereas thinner NWs exhibit broader transitions with respect to temperature. $T_{\mathrm{c}}$ for the NWs is close to that of the starting film. For the thicker $(w=20 \mathrm{~nm}) \mathrm{NWs}$, the resistance drops by an order of magnitude at liquid nitrogen temperature (77 $\mathrm{K}$ ). It drops below the measurement limit at $\sim 69 \mathrm{~K}$ (Figure $2 \mathrm{a}, \mathrm{b})$, higher than the triple point of nitrogen $(63 \mathrm{~K})$. This implies that YBCO NWs thicker than $\sim 20 \mathrm{~nm}$ could operate as fully superconducting nanoelectronic components in a pumped liquid nitrogen system. Thinner NWs have signifi- 
cantly broadened transition temperature widths, and the resistance drops to effectively zero at $\sim 20$ and $\sim 10 \mathrm{~K}$ for $w$ $=15$ and $10 \mathrm{~nm} \mathrm{NWs}$, respectively. Even these temperatures are considerably higher than those found in conventional superconductor NWs of similar widths, which are typically below liquid helium temperature $(4.2 \mathrm{~K})$. The very broad (>50 K, comparing to a few $\mathrm{K}$ for conventional superconductor NWs) transition temperature widths observed in these NWs could find applications in NW-based nano-SQUIDs ${ }^{8}$ in which the quantum-interference-induced resistance oscillations of a pair of superconductive, yet resistive, NWs are monitored for local magnetic field measurements.

The broadened transition temperature widths in the HTS NWs can be captured by the same phase-slip theories ${ }^{2,4}$ that have been applied to elemental superconductor NWs. Although phase-slip theories were originally derived for NWs with $w$ comparable to or smaller than both $\xi$ and $\lambda$, recent studies on binary compound superconductor NWs and submicrometer bridges have suggested that they may still be applicable in our case in which $\xi<w<\lambda .2,29$ For thermally activated phase slip (TAPS) processes, thermodynamic fluctuations $(\sim k T)$ instantaneously suppress superconductivity at a random point along the NW, resulting in a measurable resistance. The energy required to locally suppress superconductivity is (in SI units):

$$
\Delta F(T)=\frac{8 \sqrt{2}}{3} \frac{B_{\mathrm{c}}^{2}(T)}{2 \mu_{0}} A \xi(T)
$$

$B_{\mathrm{c}}$ is the thermodynamic critical field, and $A$ is the crosssection area of the NW. The resultant resistance then follows an Arrhenius-like equation with respect to temperature, $R_{\mathrm{TAPS}}$ $\sim \exp (-\Delta F / k T)$. Thinner NWs have smaller $A$ and therefore smaller energy barriers to overcome to acquire resistance. As a result, the resistance of thinner NWs drops more gradually below $T_{\mathrm{c}}$, and those NWs have wider transition temperature widths.

As shown in Figure $2 \mathrm{a}$, the $R-T$ relationship observed in our experiment can be satisfactorily modeled with TAPS theory using reasonable fitting parameters. The difficulty in directly applying eq 1 to obtain $\Delta F$ for data fitting lies in the fact that both $B_{\mathrm{c}}$ and $\xi$ depend on $T$ and may be different from the bulk. In previous studies on conventional superconductor NWs, Tinkham et al. derived a formula to estimate the zero-temperature limit $\Delta F(0)$ using Ginzburg-Landau relations, and $\Delta F(T)$ was then estimated using eq (1) and the relationships that $B_{\mathrm{c}} \sim\left(T_{\mathrm{c}}-T\right)$ and $\xi \sim\left(T_{\mathrm{c}}-T\right)^{-1 / 2}$ when $T \sim T_{\mathrm{c}}$, thus: ${ }^{2,30}$

$$
\begin{gathered}
\Delta F(0) \approx 0.83 \frac{L}{\xi(0)} \frac{R_{\mathrm{q}}}{R_{\mathrm{N}}} k T_{\mathrm{c}} \\
\Delta F(T) \approx \Delta F(0)\left(1-T / T_{\mathrm{c}}\right)^{3 / 2}
\end{gathered}
$$

In this way, $\Delta F$ is estimated from the known normal state resistance $R_{\mathrm{N}}$ and the NW length $L$, whereas the cross section $A$ becomes implicit in data fitting. $\xi(0)$ and $T_{\mathrm{c}}$ are fitting parameters. This approach applies only to the "dirty limit", i.e., the mean free path $l \ll$ BCS coherence length $\xi_{0}$, so $\lambda$ $\approx \lambda_{L}\left(1+\xi_{0} / l\right)^{1 / 2} \approx \lambda_{L}\left(\xi_{0} / l\right)^{1 / 2}$ (and thus $B_{\mathrm{c}}$ ) is correlated with $\boldsymbol{l}$ and therefore $R_{\mathrm{N}}$.
With $\boldsymbol{l} \sim 10 \mathrm{~nm}$ and $\xi_{0} \sim 1 \mathrm{~nm}$, HTS materials are in the opposite, "clean limit", ${ }^{13}$ so $\lambda \approx \lambda_{L}$ is not correlated with $R_{\mathrm{N}}$ and eq $2 \mathrm{a}$ is no longer applicable. For the $w=20 \mathrm{~nm}$ NWs, the transition temperature width is small $\left(T \sim T_{\mathrm{c}}\right)$, and so we have assumed a $T$-dependence of $\Delta F$ as in eq 2 b. We then used $A$ explicitly from the NW geometry, $\xi(0) \sim 2$ $\mathrm{nm}$ for YBCO from literature, ${ }^{31}$ and $B_{\mathrm{c}}(0)$ as the fitting parameter to estimate $\Delta F(0)$ from eq 1 . A reasonable fit (Figure $2 \mathrm{a}$ ) is thus obtained with $B_{\mathrm{c}}(0) \approx 0.25 \mathrm{~T}$. This value is slightly lower than the bulk YBCO value, $B_{\mathrm{c}}(0) \sim 0.5-2$ $\mathrm{T} .{ }^{31}$ This could be due to uncertainties and/or approximations in the fitting model, ${ }^{5,32}$ size effects, ${ }^{33}$ or surface effects (e.g., loss of oxygen) that reduce the effective $w$ to $<20 \mathrm{~nm}$.

For the $w=10$ and $15 \mathrm{~nm}$ arrays, the transitions are too broad and no simple analytical approximation of $\lambda(T)$ [and therefore $B_{\mathrm{c}}(T)$ ] is expected to hold over the whole range. ${ }^{4}$ On the other hand, because $w \ll \lambda, B_{\mathrm{c}}$ is correlated with the critical current density $J_{\mathrm{c}}$ by: $:^{4,34}$

$$
J_{\mathrm{c}}=a \frac{B_{\mathrm{c}}}{\mu_{0} \lambda}
$$

where $a$ is a constant of order unity, depending on the criterion used for the calculation. For example, the freeenergy criterion gives $a=1$, whereas Ginzburg-Landau theory gives $a=(2 / 3)^{3 / 2}=0.544 .^{4,34}$ Using this relationship, Tinkham demonstrated that $\Delta F(T)$ is directly proportional to the critical current $I_{\mathrm{c}}(T):{ }^{30}$

$$
\Delta F(T)=\frac{1}{a} \frac{4}{3}\left(\frac{\hbar}{2 e}\right) I_{\mathrm{c}}(T)
$$

We measured $I_{\mathrm{c}}(T)$ directly and estimated $\Delta F(T)$ by fitting to eq 4 , with $a$ as the single fitting parameter. Satisfactory fits were obtained (Figure 2a) with reasonable fitting

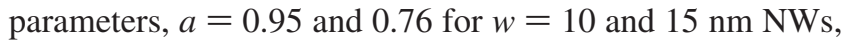
respectively. These values are quite reasonable, considering that $a$ is the only parameter used in fitting. Larger values of $a$ are expected if we take into account that the measured $I_{\mathrm{c}}$ could be less than predicted by eq 3 due to heating effects, but such effects may not be significant ${ }^{35}$ considering the very high $T_{\mathrm{c}}$ and the fact that our nanowires are epitaxially anchored to the substrate and in equilibrium with a helium gas during measurement. The deviation of the fits in the low- $T$ limit agrees with the fact that TAPS was originally developed for $T \sim T_{\mathrm{c}}$ and should not be expected to hold at $T \ll T_{\text {c }}$. We, however, cannot completely dismiss the possibility that surface impurities might contribute to the broadening of superconducting transition widths for thinner NWs.

In addition to the much higher $T_{\mathrm{c}}$, HTS NWs are also less susceptible to suppression of superconductivity from an applied magnetic field than are conventional superconductors. ${ }^{6,7}$ As shown in Figure 2b,c, a 5-T magnetic field only slightly perturbs the $R-T$ curves of the YBCO NWs. This is because HTS have higher upper critical magnetic fields compared to conventional superconductors and also our NWs have $w \ll \lambda \sim 150 \mathrm{~nm}$.

The electrical properties of the HTS NW arrays are also investigated in the high-current limit. We address whether a dissipationless supercurrent can be sustained in HTS NWs. 

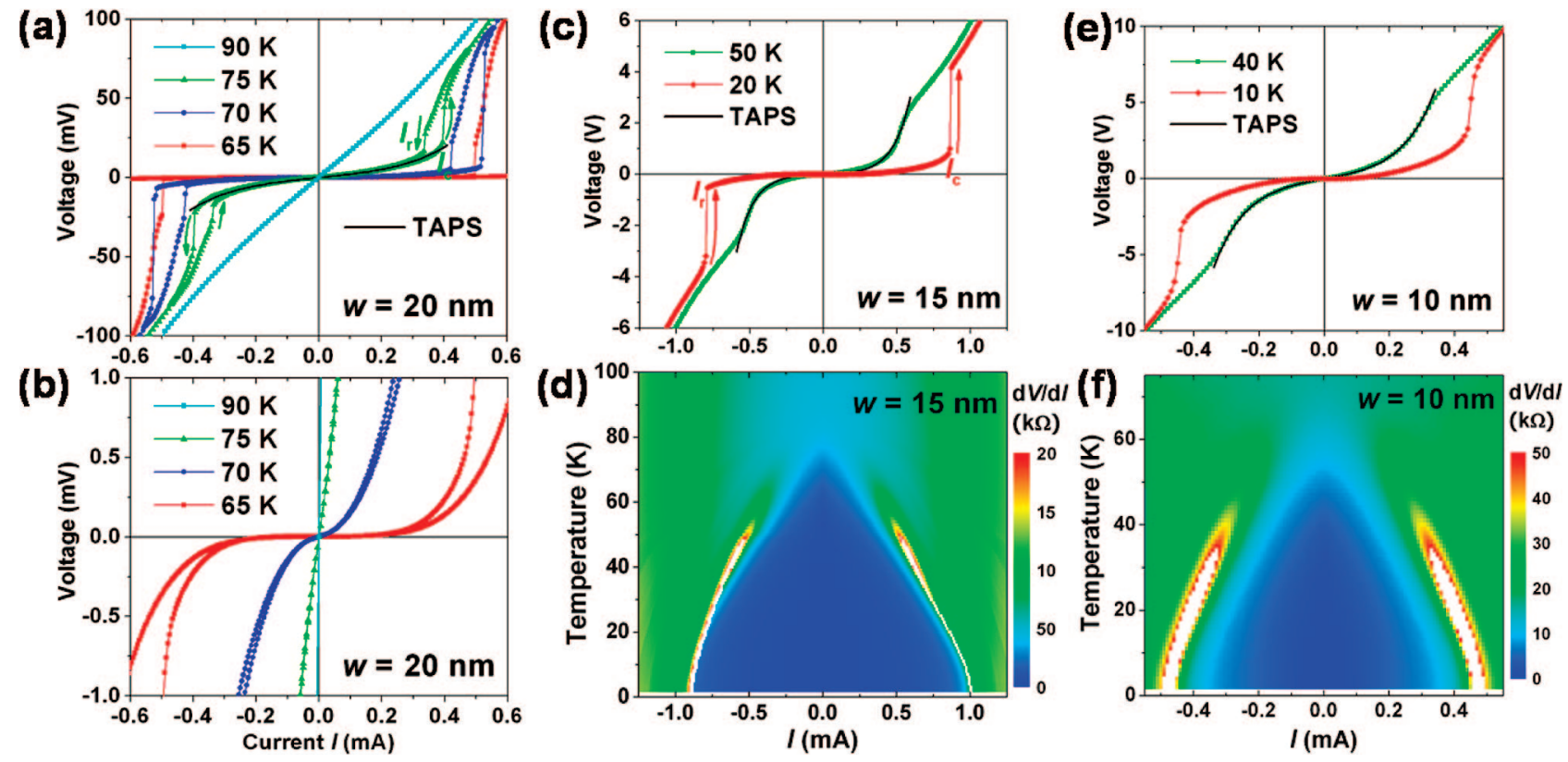

Figure 3. Electrical properties of $\mathrm{YBa}_{2} \mathrm{Cu}_{3} \mathrm{O}_{7-\delta}$ nanowire arrays in the high-current limit. (a) Four-point $V-I$ curves for an array of 400 nanowires, $w=20 \mathrm{~nm}$ and $L=5 \mu \mathrm{m}$. Measured with both increasing and decreasing current bias (arrows). $I_{\mathrm{c}}$ denotes the critical current, i.e., the highest supercurrent that can be sustained when the applied current is swept up from zero, whereas $I_{\mathrm{r}}$ denotes the retrapping current, i.e., the current level at which the array returns to the superconducting state when the current is swept back down. (b) Expansion of the plot in (a) in the low voltage range. (c,d): $V-I$ curves and $\mathrm{d} V / \mathrm{d} I$ as a function of $I$ and $T$ for an array of 150 nanowires, $w=15 \mathrm{~nm}$ and $L=$ $27 \mu \mathrm{m}$. Current sweep is from negative to positive. (e,f): $V-I$ curves and $\mathrm{d} V / \mathrm{d} I$ as a function of $I$ and $T$ for an array of 100 nanowires, $w$ $=10 \mathrm{~nm}$ and $L=50 \mu \mathrm{m}$. Black lines are TAPS fits to $V-I$ curves.

For the $w=20 \mathrm{~nm}$ NW array (Figure 3a), at $75 \mathrm{~K}$, although residual resistance is still present (cf. Figure 2a,b), a significant part of the current is already carried by supercurrent, so the voltage drop across the NWs is much smaller than when the NWs are in the normal state $(90 \mathrm{~K})$. Sweeping up from zero current, the $V-I$ relationship in this regime can be modeled with TAPS ${ }^{4,6}$ before the NWs suddenly enter the highly resistive normal state at critical current $I_{\mathrm{c}}$. When the current is swept down, the NWs return to the superconducting state at a lower current level, $I_{\mathrm{r}}$. Such hysteresis has been reported in conventional superconductor NWs. ${ }^{71,156} \mathrm{At}$ lower $T$, a larger portion of the current is carried by the supercurrent, resulting in smaller voltage drops, and $I_{\mathrm{c}}$ increases steadily. At $70 \mathrm{~K}$, where the low-current-limit resistance is very close to zero (cf. Figure 2a,b), dissipationless (characterized by a zero voltage drop) supercurrent starts to emerge (Figure 3b). At $65 \mathrm{~K}$, which is still higher than the triple point of nitrogen $(63 \mathrm{~K})$, the low-currentlimit resistance has dropped below a measureable value and a dissipationless supercurrent is sustained up to $\sim 0.2 \mathrm{~mA}$.

Similar trends are found for the thinner NWs (Figure 3c,e). Dissipationless supercurrent occurs below the temperature at which the low-current-limit resistance drops to effectively zero (red curves), which is $\sim 20$ and $\sim 10 \mathrm{~K}$ for $w=15$ and $10 \mathrm{~nm}$ NWs, respectively. These temperatures are still considerably higher than conventional superconductor NWs. As with NWs of conventional superconductors, ${ }^{7,36}$ hysteresis in the $V-I$ curves is less prominent for thinner NWs.

The wide superconducting transition temperature width found in the thinner NWs is further studied by plotting the differential resistance $\mathrm{d} V / \mathrm{d} I$ as a function of $I$ and $T$ (Figure 3d,f). At $T<T_{\mathrm{c}}$, a gap of low differential resistance opens up at low current due to supercurrent, and the width of this gap $\left(I_{\mathrm{c}}+I_{\mathrm{r}}\right)$ increases steadily with decreasing temperature. Transitions between the superconducting and normal states sharpen, and regions with effectively zero differential resistance start to appear at finite current. In contrast to arrays of conventional superconductor NWs, no peak lines or branching features are observed in the $\mathrm{d} V / \mathrm{d} I-T$ plots. Similarly clean and featureless $\mathrm{d} V / \mathrm{d} I$ plots were obtained for all YBCO NW devices. Peak line features in $\mathrm{d} V / \mathrm{d} I-T$ plots are typical for long, conventional superconductor NWs, ${ }^{7,15}$ reflecting multiple voltage-jump steps in $V-I$ curves. Each such voltage-jump step is associated with the emergence of a localized resistive "phase-slip center" (PSC) along the NW. ${ }^{4}$ Each PSC occupies a characteristic length of $\sim 2 \Lambda$, where $\Lambda$ is the quasiparticle diffusion length $(\sim 10$ $\mu \mathrm{m}$ for elemental superconductors). Theory predicts that for a uniform filament of length $L$, approximately $L / 2 \Lambda$ steps should occur between $I_{\mathrm{c}}$ and $2 I_{\mathrm{c}},{ }^{4,37}$ and we have recently verified this in $\mathrm{Nb} \mathrm{NW}$ arrays. ${ }^{7}$ HTS materials have extremely small $\Lambda\left(<\sim 10 \mathrm{~nm}\right.$ for $\left.\mathrm{YBCO}^{38}\right)$, so all the NWs investigated in this work satisfy $L \gg 2 \Lambda$, and in this limit PSC steps are not expected to be resolvable. ${ }^{37}$ Voltage-jump steps have been previously observed in several studies on HTS micrometer and submicrometer bridges, ${ }^{21,22,39}$ but they are not obviously a consequence of PSCs. ${ }^{21}$

High-temperature superconductivity is retained in NWs with widths as small as $10 \mathrm{~nm}$ and lengths up to $200 \mu \mathrm{m}$, with an onset $T_{\mathrm{c}}$ that is higher than liquid nitrogen temperature. The $w=20 \mathrm{~nm}$ NWs become fully superconducting and are capable of carrying dissipationless current at pumped 
liquid nitrogen temperatures. For thinner NWs, the transition temperature widths are significantly broadened, and complete superconductivity is obtained at $\sim 20$ and $\sim 10 \mathrm{~K}$ for $w=$ 15 and $10 \mathrm{~nm}$ NWs, respectively. The results are modeled satisfactorily using phase-slip theories that generate reasonable parameters. These NWs can function as superconducting nanoelectronic components with broader working temperature ranges as compared to conventional superconductor NWs. A possible unique application of these NWs, as near perfect current limiters, is discussed in the Supporting Information.

Acknowledgment. We thank Yue Zou for helpful discussions. This work was supported by the Department of Energy (DE-FG02-04ER46175).

Supporting Information Available: Description of the fabrication processes. Discussions on HTS NW as a current limiter. This material is available free of charge via the Internet at http://pubs.acs.org.

\section{References}

(1) Bezryadin, A.; Lau, C. N.; Tinkham, M. Nature 2000, 404, 971.

(2) Lau, C. N.; Markovic, N.; Bockrath, M.; Bezryadin, A.; Tinkham, M. Phys. Rev. Lett. 2001, 87, 217003.

(3) Zgirski, M.; Riikonen, K. P.; Touboltsev, V.; Arutyunov, K. Nano Lett. 2005, 5, 1029.

(4) Tinkham, M. Introduction to Superconductivity, 2nd ed.; McGrawHill: New York, 1996.

(5) Tian, M. L.; Wang, J. G.; Kurtz, J. S.; Liu, Y.; Chan, M. H. W.; Mayer, T. S.; Mallouk, T. E. Phys. Rev. B 2005, 71, 104521.

(6) Altomare, F.; Chang, A. M.; Melloch, M. R.; Hong, Y. G.; Tu, C. W. Phys. Rev. Lett. 2006, 97, 017001.

(7) Xu, K.; Heath, J. R. Nano Lett. 2008, 8, 136.

(8) Hopkins, D. S.; Pekker, D.; Goldbart, P. M.; Bezryadin, A. Science 2005, 308, 1762.

(9) Makhlin, Y.; Schon, G.; Shnirman, A. Nature 1999, 398, 305.

(10) Nakamura, Y.; Pashkin, Y. A.; Tsai, J. S. Nature 1999, 398, 786.

(11) Mooij, J. E.; Nazarov, Y. V. Nat. Phys. 2006, 2, 169.

(12) Divochiy, A.; Marsili, F.; Bitauld, D.; Gaggero, A.; Leoni, R.; Mattioli, F.; Korneev, A.; Seleznev, V.; Kaurova, N.; Minaeva, O.; Gol'tsman, G.; Lagoudakis, K. G.; Benkhaoul, M.; Levy, F.; Fiore, A. Nat. Photonics 2008, 2, 302.

(13) Burns, G. High-Temperature Superconductivity: An Introduction; Academic Press: Boston, 1992.
(14) Wu, Y. Y.; Messer, B.; Yang, P. D. Adv. Mater. 2001, 13, 1487.

(15) Falk, A.; Deshmukh, M. M.; Prieto, A. L.; Urban, J. J.; Jonas, A.; Park, H. Phys. Rev. B 2007, 75, 020501(R).

(16) Hall, S. R. Adv. Mater. 2006, 18, 487.

(17) Zhang, G. Q.; Lu, X. L.; Zhang, T.; Qu, J. F.; Wang, W.; Li, X. G.; Yu, S. H. Nanotechnology 2006, 17, 4252.

(18) Jiang, H.; Huang, Y.; How, H.; Zhang, S.; Vittoria, C.; Widom, A.; Chrisey, D. B.; Horwitz, J. S.; Lee, R. Phys. Rev. Lett. 1991, 66, 1785.

(19) Vanderharg, A. J. M.; Vanderdrift, E.; Hadley, P. IEEE Trans. Appl. Supercond. 1995, 5, 1448.

(20) Larsson, P.; Nilsson, B.; Ivanov, Z. G. J. Vac. Sci. Technol., B 2000, $18,25$.

(21) Bonetti, J. A.; Caplan, D. S.; Van Harlingen, D. J.; Weissman, M. B. Phys. Rev. Lett. 2004, 93, 087002.

(22) Mikheenko, P.; Deng, X.; Gildert, S.; Colclough, M. S.; Smith, R. A.; Muirhead, C. M.; Prewett, P. D.; Teng, J. Phys. Rev. B 2005, 72, 174506.

(23) Melosh, N. A.; Boukai, A.; Diana, F.; Gerardot, B.; Badolato, A.; Petroff, P. M.; Heath, J. R. Science 2003, 300, 112.

(24) Green, J. E.; Choi, J. W.; Boukai, A.; Bunimovich, Y.; JohnstonHalperin, E.; Delonno, E.; Luo, Y.; Sheriff, B. A.; Xu, K.; Shin, Y. S.; Tseng, H. R.; Stoddart, J. F.; Heath, J. R. Nature 2007, 445, 414.

(25) Wang, D. W.; Sheriff, B. A.; Heath, J. R. Nano Lett. 2006, 6, 1096.

(26) Ban, M.; Takenaka, T.; Hayashi, K.; Suzuki, K.; Enomoto, Y. Jpn. J. Appl. Phys., Part 1 1996, 35, 4318.

(27) Oka, K.; Iri, T. Jpn. J. Appl. Phys., Part 1 1992, 31, 2689.

(28) Wang, D. W.; Bunimovich, Y.; Boukai, A.; Heath, J. R. Small 2007, 3, 2043.

(29) Bell, M.; Sergeev, A.; Mitin, V.; Bird, J.; Verevkin, A.; Gol'tsman, G. Phys. Rev. B 2007, 76, 094521 .

(30) Tinkham, M.; Lau, C. N. Appl. Phys. Lett. 2002, 80, 2946.

(31) Poole, C. P. Handbook of Superconductivity; Academic Press: San Diego, CA, 2000.

(32) Giordano, N. Phys. Rev. B 1990, 41, 6350.

(33) Anlage, S. M.; Sze, H.; Snortland, H. J.; Tahara, S.; Langley, B.; Eom, C. B.; Beasley, M. R.; Taber, R. Appl. Phys. Lett. 1989, 54, 2710.

(34) Bardeen, J. Rev. Mod. Phys. 1962, 34, 667.

(35) Rogachev, A.; Bezryadin, A. Appl. Phys. Lett. 2003, 83, 512.

(36) Tinkham, M.; Free, J. U.; Lau, C. N.; Markovic, N. Phys. Rev. B 2003, 68, 134515.

(37) Tinkham, M. J. Low Temp. Phys. 1979, 35, 147.

(38) Yeh, N. C.; Vasquez, R. P.; Fu, C. C.; Samoilov, A. V.; Li, Y.; Vakili, K. Phys. Rev. B 1999, 60, 10522.

(39) Jelila, F. S.; Maneval, J. P.; Ladan, F. R.; Chibane, F.; Marie-deFicquelmont, A.; Mechin, L.; Villegier, J. C.; Aprili, M.; Lesueur, J. Phys. Rev. Lett. 1998, 81, 1933.

NL802264X 